\title{
The evolving spectrum of the planetary nebula Hen 2-260^
}

\author{
M. Hajduk ${ }^{1}$, P. A. M. van $\operatorname{Hoof}^{2}$, K. Gesicki ${ }^{3}$, A. A. Zijlstra ${ }^{4}$, S. K. Górny ${ }^{1}$, and M. Gładkowski ${ }^{1}$ \\ ${ }^{1}$ Nicolaus Copernicus Astronomical Center, ul. Rabiańska 8, 87-100 Toruń, Poland \\ e-mail: Marcin.Hajduk@astri.uni.torun.pl \\ 2 Royal Observatory of Belgium, Ringlaan 3, 1180 Brussels, Belgium \\ 3 Centre for Astronomy, Faculty of Physics, Astronomy and Informatics, Nicolaus Copernicus University, Grudziadzka 5, \\ 87-100 Torun, Poland \\ 4 Jodrell Bank Centre for Astrophysics, Alan Turing Building, Manchester M13 9PL, UK
}

Received 24 September 2013 / Accepted 20 March 2014

\begin{abstract}
Aims. We analysed the planetary nebula Hen 2-260 using optical spectroscopy and photometry. We compared our observations with the data from literature to search for evolutionary changes. We also searched for photomertic variability of the central star. Methods. The object Hen 2-260 was observed with the SAAO $1.0 \mathrm{~m}$ telescope (photometry) and the SALT telescope (low resolution spectroscopy). We also used archival high resolution Very Large Telescope spectra and Hubble Space Telescope imaging. The nebular line fluxes were modelled with the Cloudy photoionization code to derive the stellar and nebular parameters.

Results. The planetary nebula shows a complex structure and possibly a bipolar outflow. The nebula is relatively dense and young. The central star is just starting $\mathrm{O}^{+}$ionization $\left(T_{\mathrm{eff}} \approx 30000 \mathrm{~K}\right)$. Comparison of our observations with literature data indicates a $50 \%$ increase of the [O III] $5007 \AA$ line flux between 2001 and 2012. We interpret it as the result of the progression of the ionization of $\mathrm{O}^{+}$. The central star evolves to higher temperatures at a rate of $45 \pm 7 \mathrm{~K} \mathrm{yr}^{-1}$. The heating rate is consistent with a final mass of $0.626_{-0.005}^{+0.003} M_{\odot}$ or $0.645_{-0.008}^{+0.008} M_{\odot}$ for two different sets of post-AGB evolutionary tracks from literature. The photometric monitoring of Hen 2-260 revealed variations on a timescale of hours or days. There is no direct indication for central star binarity in the spectrum nor for a strong stellar wind. The variability may be caused by pulsations of the star.

Conclusions. The temperature evolution of the central star can be traced using spectroscopic observations of the surrounding planetary nebula spanning a timescale of roughly a decade. This allows us to precisely determine the stellar mass, since the pace of the temperature evolution depends critically on the core mass. The method is independent of the absolute age of the nebula. The kinematical age of the nebula is consistent with the age obtained from the evolutionary track. The final mass of the central star is close to the mass distribution peak for central stars of planetary nebulae found in other studies. The object belongs to a group of young central stars of planetary nebulae showing photometric variability.
\end{abstract}

Key words. stars: AGB and post-AGB - stars: evolution - planetary nebulae: general - planetary nebulae: individual: Hen 2-260

\section{Introduction}

Planetary nebulae (PNe) are the final stage in evolution of stars with initial masses of $1-8 M_{\odot}$. Low-mass stars lose most of their material via a stellar wind during the asymptotic giant branch (AGB) phase. Once the star has shed its envelope, only a hot core surrounded by a tiny $\left(10^{-4} M_{\odot}\right)$ hydrogen envelope remains. The star subsequently moves towards the white dwarf regime in the Herzsprung-Russell diagram, where the thermonuclear reactions finally stop.

The pace of evolution critically depends on the final mass of the central star of a PN. The star reaches its maximum temperature on a timescale of several decades for the most massive objects up to several thousands years for low mass central stars (Blöcker 1995). On its path towards high effective temperatures, the central star passes through the instability strip. Pulsational instabilities in post-AGB stars have been studied by Zalewski (1993) and Gautschy (1993). Several examples of variable young central stars of PNe have been found by

* Table 4 is only available at the CDS via anonymous ftp to cdsarc.u-strasbg.fr (130.79.128.5) or via http://cdsarc.u-strasbg.fr/viz-bin/qcat?]/A+A/567/A15
Handler (2003). Variability was attributed to either stellar wind instabilities or pulsations.

Only in a few cases, a change of the ionizing flux of a central star was observed on a timescale of years or decades. Feibelman et al. (1992) reported a variability of emission lines in the PNe IC 4997 and NGC 6572. More recently, Arkhipova et al. (2013) discovered the variability of the PN Hen 3-1357 and its central star. In the case of NGC 7027, observations at radio frequencies for 25 years allowed for the determination of the central star mass (Zijlstra et al. 2008).

\section{PN Hen $2-260$}

The planetary nebula Hen 2-260 (PN G 008.2+06.8) is located 10 degrees away from the Galactic Center. Hultzsch et al. (2007) modelled the central star of Hen 2-260 with a H-rich model atmosphere with $T_{\text {eff }}=28000 \pm 1500 \mathrm{~K}$. No stellar emission lines were observed, which indicated a low mass loss rate. Hultzsch et al. (2007) derived a spectroscopic distance to the nebula of $11.2_{-1.6}^{+2.0} \mathrm{kpc}$. Most of the statistical determinations of the distance in the literature for Hen 2-260 are consistent with this result (e.g., $12.17 \mathrm{kpc}$ derived by Zhang 1995; $11.44 \mathrm{kpc}$ by 
Van de Steene \& Zijlstra 1994; $17.0 \pm 3.4$ kpc by Stanghellini \& Haywood 2010).

The dust shows the presence of amorphous and crystalline silicates (Perea-Calderón et al. 2009; Górny et al. 2010). Guzman-Ramirez et al. (2011) discovered very faint PAH emission at $8.6 \mu \mathrm{m}$.

An excitation class of 0.5 was given to this object by Escudero et al. (2004). They determined the (reddened) [O III] $5007 \AA$ A line flux to be only 5.1 (Table 1). Acker et al. (1991) did not observe the [O III] $5007 \AA$ nebular line in the spectrum taken almost two decades before.

\section{Observations}

The photometric observations were done at the SAAO observatory using the $1.0 \mathrm{~m}$ telescope in five periods between 2010 August 25 - September 1, 2011 March 24-29, 2011 May 4-10, 2011 August 24-30, and 2012 May 9-22 in the $I$ filter. We used the SITe CCD camera, which measures $1024 \times 1024$ pixels with the field of view of about 5 arcmin squared.

The Hubble Space Telescope (HST) snapshot survey of bulge PNe was held in 2002 and 2003 (proposal 9356, PI Albert Zijlstra). The images were obtained using the Wide Field Planetary Camera in the F656N (two $100 \mathrm{~s}$ exposures), F502N (two $230 \mathrm{~s}$ exposures), and $F 547 M$ (one $60 \mathrm{~s}$ exposure) filters on 2003 March 13.

High-resolution spectra were obtained with the Very Large Telescope (VLT) on 2005 April 21 with the Ultraviolet and Visual Echelle Spectrograph (UVES) in three wavelength ranges: $3280-4480,4600-5600$, and 5660-6660 $\AA$ with the spectral resolution of about $\lambda / \Delta \lambda=60000$. Three $600 \mathrm{~s}$ exposures were made. The slit was 11 arcsec long and $0.5 \operatorname{arcsec}$ wide. The spectrum was flux calibrated using a standard star observation. The line flux uncertainty derived from the comparison of the fluxes of the same lines measured in individual spectra is about $2 \%$ for the strong lines and $6 \%$ for the lines $\leq 1 \%$ of the $\mathrm{H} \beta$ flux.

The SALT low-resolution spectra were obtained on 2012 May 29 with the $9001 / \mathrm{mm}$ grating. The 1 arcsec wide and 8 arcmin long slit was used. The spectral coverage was 4350-7400 ̊ with a spectral resolution of about 6000 at the central wavelength. Two spectra were taken with exposure times of 120 and $900 \mathrm{~s}$. Most of the fluxes used in this work were derived from the $900 \mathrm{~s}$ spectrum, except for the $\mathrm{H} \alpha, \mathrm{H} \beta,[\mathrm{N} \mathrm{II}] 6548 \AA$, [N II] $6584 \AA$, [O II] $7320 \AA$ and [O II] $7330 \AA$ lines. These lines were saturated in the $900 \mathrm{~s}$ spectrum and their fluxes were measured in the $120 \mathrm{~s}$ spectrum.

The spectra were flux calibrated using the standard star observation taken during the same night. Line fluxes were measured from both spectra and compared. The line flux uncertainty derived from the comparison of 120 and $900 \mathrm{sec}$ spectra is about $5 \%$ for the strong lines, $10 \%$ for the lines $\leq 1 \%$ of the $\mathrm{H} \beta$ flux, and $25 \%$ for $\leq 0.2 \%$ of the $\mathrm{H} \beta$ flux.

The Radcliffe $1.9 \mathrm{~m}$ telescope low-resolution spectra were obtained using the grating spectrograph with a 1.5 arcsec slit between 2012 May 5 and 9 . The total exposure time was about $3 \mathrm{~h}$. The wavelength coverage is from 3625 to $7600 \AA$ and the spectral resolution of about 2500 at the central wavelength. The spectra were flux calibrated. A different set of flux standards was measured during each night. The uncertainty of the fluxes is about $5 \%$, measured from the spectra collected in different nights.
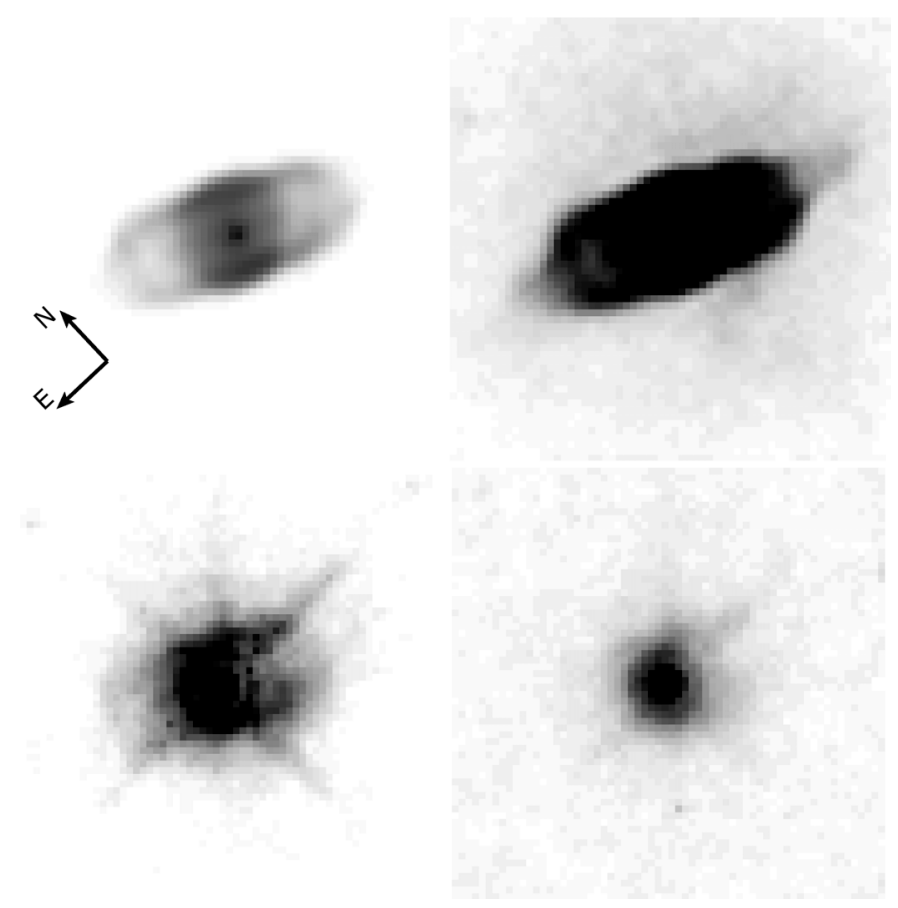

Fig. 1. Upper row: HST F656N deconvolved image of the PN Hen 2260. Different cut levels were applied to enhance the bipolar structure (left) and bipolar outflow (right). Lower row: HST F547M (left) and F502N (right) deconvolved images. All the images are 3 arcsec wide. Logarithmic intensity scale was applied to all the images.

Some of the nebular fluxes may be affected by the underlying stellar absorptions. This applies to faint He I absorption lines in the low-resolution spectra. The He I fluxes may be systematically higher in the VLT spectrum than in the low resolution spectra.

The data were reduced using the IRAF package. We used the ISIS difference image analysis package to obtain magnitudes. This allows us to avoid the instrumental effects caused by the presence of a resolved nebula. Otherwise, the nebular contribution may degrade the quality of the lightcurve.

To search for periodic variability, we used the PERIOD04 program (Lenz \& Breger 2005) and the analysis of variance (ANOVA) method (Schwarzenberg-Czerny 1996).

\section{Results}

\subsection{HST imaging}

The HST image of Hen 2-260 reveals a complex morphology of the object. The brightest, central part of the nebula appears to be roughly spherical. An outer, elongated limb-brightened structure appears to be open near the polar axes (Fig. 1). A polar outflow appears to be present in the $F 656 N(\mathrm{H} \alpha)$ filter image. The maximum extent of the nebula is $1.8 \times 0.85$ arcsec in the $F 656 N$ filter image.

The central star is clearly visible in the F547M (continuum) and $F 502 N$ ([O III] $5007 \AA$ ) filters. The $V$-band magnitude of the central star, measured using the $F 547 M$ filter image, is $14.13 \pm 0.03$. We observe a faint, extended nebular component in the $F 547 M$ filter image (and probably in $F 502 N$, too) after the deconvolution was performed with the point spread function (PSF) that was generated with the TinyTim tool (Krist et al. 2011). However, a residual PSF pattern (diffration spikes and the Airy rings) is clearly present in the images and hampers the analysis of the $F 547 M$ and $F 502 N$ filter images. The brightest parts 
Table 1. Comparison of fluxes of the strongest nebular lines.

\begin{tabular}{|c|c|c|c|c|c|c|}
\hline$\lambda[\AA]$ & Ion & $\begin{array}{c}\text { ESO } 1.52 \mathrm{~m}^{a} \\
1984-04-30 \\
\text { JD 2 445 } 820 \\
\end{array}$ & $\begin{array}{c}\text { ESO } 1.52 \mathrm{~m}^{b} \\
2001-07-01 \\
\text { JD 2 452 091 } \\
\end{array}$ & $\begin{array}{c}\text { VLT } \\
\text { 2005-04-20 } \\
\text { JD 2453 480 }\end{array}$ & $\begin{array}{l}\text { SAAO } 1.9 \mathrm{~m} \\
2012-05-07 \\
\text { JD } 2456054\end{array}$ & $\begin{array}{c}\text { SALT } \\
\text { 2012-05-29 } \\
\text { JD 2 456076 }\end{array}$ \\
\hline 4861 & $\mathrm{HI}$ & $100.0 \pm 5.0$ & $100.0 \pm 2.7$ & $100.0 \pm 1.0$ & $100.0 \pm 10.0$ & $100.0 \pm 5.0$ \\
\hline 4959 & {$[\mathrm{O} \mathrm{III}]$} & - & $1.6 \pm 0.2$ & $1.8 \pm 0.1$ & $2.6 \pm 0.3$ & $2.4 \pm 0.3$ \\
\hline 5007 & [O III] & - & $5.1 \pm 0.4$ & $5.5 \pm 0.1$ & $7.8 \pm 0.8$ & $7.9 \pm 0.4$ \\
\hline 5754 & {$[\mathrm{~N}$ II $]$} & $3.0 \pm 1.0$ & $4.1 \pm 0.4$ & $4.1 \pm 0.1$ & $4.1 \pm 0.5$ & $4.2 \pm 0.3$ \\
\hline 5875 & $\mathrm{He} \mathrm{I}$ & $4.0 \pm 1.0$ & $2.1 \pm 0.2$ & $2.8 \pm 0.1$ & $2.9 \pm 0.3$ & $2.9 \pm 0.2$ \\
\hline 6300 & {$[\mathrm{OI}]$} & $5.0 \pm 1.0$ & $3.5 \pm 0.3$ & $3.4 \pm 0.1$ & $3.7 \pm 0.4$ & $3.4 \pm 0.2$ \\
\hline 6312 & [S III] & - & $1.3 \pm 0.2$ & $1.4 \pm 0.1$ & $2.0 \pm 0.2$ & $1.5 \pm 0.1$ \\
\hline 6364 & {$[\mathrm{O} \mathrm{I}]$} & $2.2 \pm 0.7$ & $1.1 \pm 0.2$ & $1.1 \pm 0.1$ & $1.1 \pm 0.2$ & $1.1 \pm 0.1$ \\
\hline 6548 & {$[\mathrm{~N} \mathrm{II}]$} & $77.0 \pm 4.0$ & $59.6 \pm 1.9$ & $57.8 \pm 0.6$ & $61.8 \pm 6.2$ & $62.9 \pm 3.2$ \\
\hline 6563 & H I & $581.0 \pm 30.0$ & $498.9 \pm 7.7$ & $509.0 \pm 5.0$ & $493.0 \pm 50.0$ & $493.0 \pm 25.0$ \\
\hline 6584 & {$[\mathrm{~N} \mathrm{II}]$} & $213.0 \pm 11.0$ & $183.8 \pm 4.0$ & $190.0 \pm 2.0$ & $179.0 \pm 18.0$ & $186.0 \pm 10.0$ \\
\hline 6678 & $\mathrm{He} \mathrm{I}$ & - & $0.5 \pm 0.1$ & & $1.4 \pm 0.2$ & $0.8 \pm 0.1$ \\
\hline 6716 & [S II] & $4.0 \pm 1.0$ & $4.2 \pm 0.4$ & & $4.2 \pm 0.5$ & $3.6 \pm 0.4$ \\
\hline 6731 & [S II] & $10.0 \pm 1.0$ & $9.0 \pm 0.6$ & & $8.0 \pm 0.8$ & $8.1 \pm 0.5$ \\
\hline 7002 & {$[\mathrm{OI}]$} & - & $0.9 \pm 0.2^{c}$ & & $0.9 \pm 0.1$ & $0.7 \pm 0.1$ \\
\hline 7065 & $\mathrm{He} \mathrm{I}$ & - & $1.1 \pm 0.2$ & & $1.9 \pm 0.2$ & $1.9 \pm 0.1$ \\
\hline 7135 & [Ar III] & - & $1.6 \pm 0.2$ & & $2.1 \pm 0.3$ & $2.3 \pm 0.2$ \\
\hline 7320 & {$[\mathrm{O}$ II $]$} & & $35.8 \pm 1.5$ & & $44.8 \pm 2.3$ & $49.8 \pm 2.5$ \\
\hline 7330 & [O II] & $0.0 \pm 4.0$ & $41.2 \pm 1.8$ & & $39.5 \pm 2.0$ & $40.4 \pm 2.1$ \\
\hline
\end{tabular}

Notes. ${ }^{(a)}$ Acker et al. (1991). ${ }^{(b)}$ Escudero et al. (2004). ${ }^{(c)}$ misidentified as [Ar V] $\lambda 7005.6$ by Escudero et al. (2004).

of the nebula in the $\mathrm{H} \alpha$ image are located at a projected distance of about 0.2 arcsec away from the central star, which is similar to the radius of to the 1st Airy ring.

We obtained $\log F(\mathrm{H} \alpha)=-11.19 \pm 0.03 \mathrm{erg} \mathrm{cm}^{-2} \mathrm{~s}^{-1}$ from the HST image, using the calibration formulae given by Dudziak \& Walsh (1997). The $\mathrm{H} \alpha$ line equivalent width is about $-670 \AA$, while the $F 656 N$ filter width is $21.5 \AA$. Thus, the continuum contribution to the $F 656 \mathrm{~N}$ inband flux is only about $3 \%$.

We obtained an [O III] $5007 \AA$ to $\mathrm{H} \alpha$ line flux ratio of 0.07 from the HST images. The [O III] $5007 \AA$ to $\mathrm{H} \alpha$ line flux ratio obtained from the SALT spectrum is about 0.016. The discrepancy is due to the continuum contribution to the $F 502 \mathrm{~N}$ filter flux. The equivalent width of the [O III] $5007 \AA$ line is $-8 \AA$ (measured from the SALT spectrum), while the F502N filter width is $26.9 \AA$. The contribution of the continuum to the flux in the $F 502 \mathrm{~N}$ filter is expected to be 3.4 times higher than the [O III] $5007 \AA$ line flux. The contribution of the [Fe III] $5011 \AA$ and $\mathrm{HeI} 5016 \AA$ flux to the inband flux in the $F 502 N$ filter is about $10 \%$ of the [O III] $5007 \AA$ line flux.

\subsection{Optical spectra}

We obtained the extinction of $\mathrm{c}(\mathrm{H} \beta)=0.69 \pm 0.09$ by using the $\mathrm{H} \alpha$ to $\mathrm{H} \beta$ line flux ratio and by assuming the standard value of the total to selective absorption ratio $R_{V}=\frac{A_{V}}{E(B-V)}$ of 3.1 . The optical spectra were dereddened using the extinction curve given by Fitzpatrick (1999).

The ratio of the radio continuum flux at $5 \mathrm{GHz}$ and the observed $\mathrm{H} \beta$ flux (both quantities are given in Table 2) can also be used to obtain the extinction (e.g., Pottasch 1984). The predicted (dereddened) $\log F(\mathrm{H} \beta)$ flux is $-11.37 \mathrm{erg} \mathrm{cm}^{-2} \mathrm{~s}^{-1}$, which gives $c(\mathrm{H} \beta)$ of $0.76 \pm 0.11$.

Pottasch \& Bernard-Salas (2013) obtained the extinction $c(\mathrm{H} \beta)$ of 1.06 by using the $\mathrm{H}(6-5) 7.46 \mu \mathrm{m}$ and $\mathrm{H}(7-6) 12.37 \mu \mathrm{m}$ line fluxes. They suggested that the discrepancy between the extinction obtained by using radio continuum flux and the infrared hydrogen line fluxes could result from the non-negligible optical depth at radio frequencies. However, Hen 2-260 has a brightness temperature of only $920 \mathrm{~K}$ at $5 \mathrm{GHz}$ (Aaquist \& Kwok 1990). Using the emission measure computed in the Cloudy model, we determined the optical thickness of $\tau_{1.4 \mathrm{GHz}}=0.45$ and $\tau_{5 \mathrm{GHz}}=0.03$. The observed fluxes of the radio spectrum are consistent with those results. The agreement of the extinction determinations using the optically thin $5 \mathrm{GHz}$ with the extinction from the Balmer decrement validates our choice.

The nebular line fluxes are shown in Table 3 (SALT and $1.9 \mathrm{~m}$ Radcliffe telescope spectra) and Table 4 (VLT spectrum). We compared the observed and theoretical ratios of the pairs of lines coming from the same upper levels to assess the reliability of the error determinations of the nebular fluxes. The transition probabilities were taken from the following: Nahar \& Pradhan (1996) for [Fe III] lines, Quinet et al. (1996) for [Fe II] lines and Storey \& Zeippen (2000) for [O III] 5007/4959 А, [N II] 6584/6548 $\AA$ and [O I] 6300/6364 line ratios. The $\mathrm{Si}$ II transition probabilities were taken from the Atomic Line List ${ }^{1}$.

The average $\chi^{2}$ was 0.38 for the six ratios determined from the SALT spectrum and 1.32 for the VLT spectrum for the nineteen ratios. We assumed a $20 \%$ uncertainty for the transition probabilities for the [Fe III] lines, $15 \%$ uncertainty for the [Fe II] lines, $6 \%$ to $15 \%$ uncertainty for the Si II lines, and negligible uncertainty for the remaining lines. The main sources of the high value of $\chi^{2}$ derived for the VLT spectrum were the [N II] $6584 / 6548 \AA$ line flux ratio and the Si II 5958/5979 A line ratio. The $6584 \AA$ line flux can be affected by uncertain calibration close to the edge of the wavelength range. The Si II $5958 \AA$ line flux can be affected by the nearby $\mathrm{O}$ I emission. We obtained an averaged $\chi^{2}$ of 0.80 for seventeen remaining flux ratios. Good agreement between the theoretical and observed flux ratios confirms that we derived reliable uncertainties for the fluxes.

We compared the fluxes derived with the SALT telescope with the fluxes published by Escudero et al. (2004). Some of the fluxes agree very well (Table 1). However, [O III], He I, [Ar III]

1 http://www.pa.uky.edu/ peter/newpage/ 

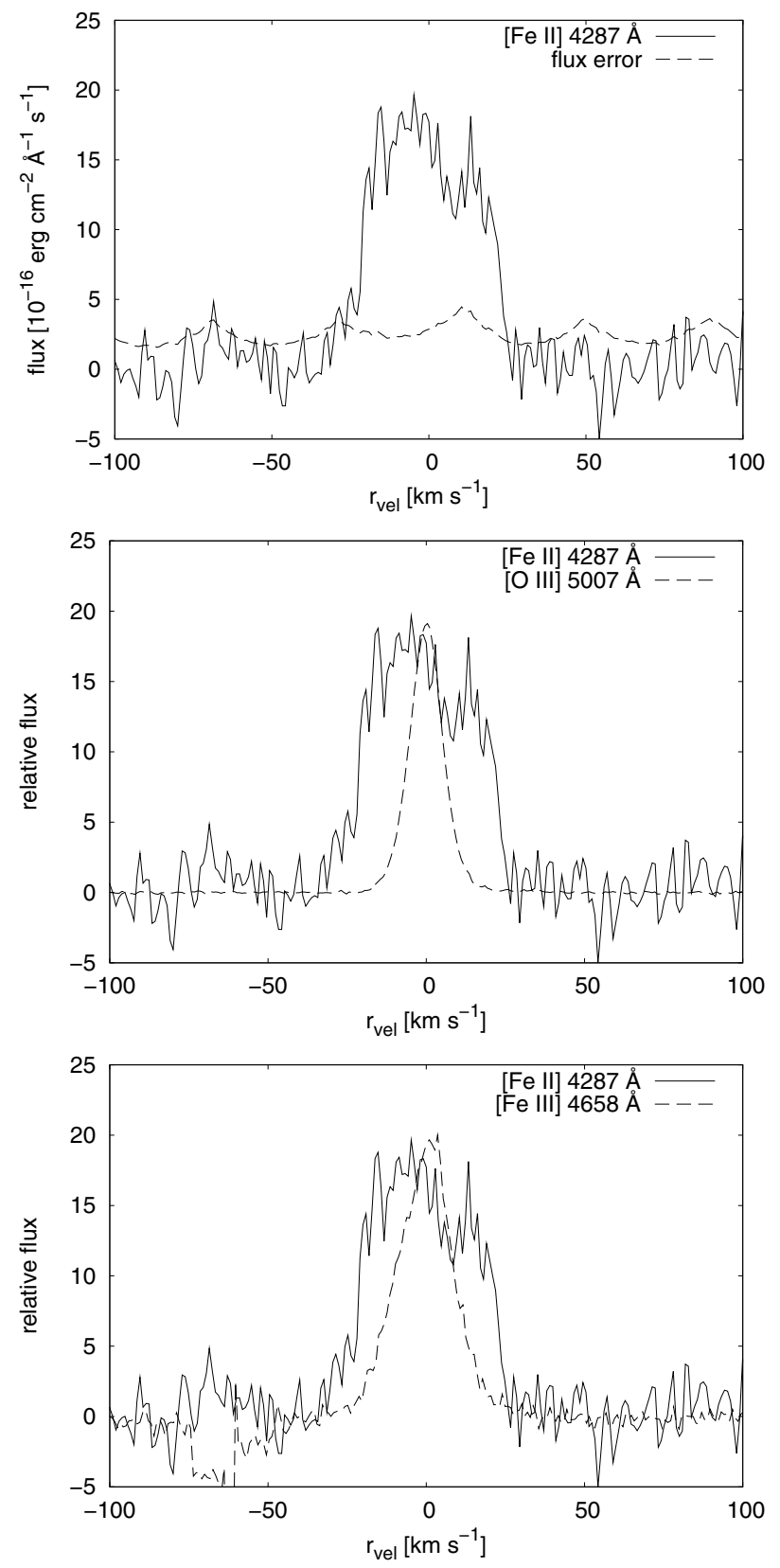

Fig. 2. VLT continuum subtracted spectrum of the [Fe II] $4287 \AA$ line and flux error (upper), [Fe II] $4287 \AA$ and [O III] $5007 \AA$ lines (middle), and [Fe II] $4287 \AA$ and [Fe III] $4658 \AA$ lines (lower).

and [S III] fluxes seem to have increased between 2001 and 2012. In particular, we obtained a [O III] $5007 \AA$ line flux of $7.9 \pm 0.4$ (relative to $F(\mathrm{H} \beta)=100$ ) using the SALT spectrum, which is markedly higher than previous observers. Escudero et al. (2004) obtained 5.1 \pm 0.4 , while Acker et al. (1991) do not report a detection of this line. The flux increase may indicate the increasing stellar temperature.

One can question if the observed increase in the [O III] $5007 \AA$ flux is not of instrumental origin. The spectra in different epochs were obtained with different instruments and configurations. In particular, Escudero et al. (2004) used a 2 arcsec wide slit; a 1 arcsec slit was used in the SALT spectrum; a 1.5 arcsec slit for the $1.9 \mathrm{~m}$ Radcliffe telescope spectrum; and the VLT spectrum was obtained with a 0.5 arcsec slit.

The spectra obtained more recently could be more sensitive to the inner regions of the nebula compared to the spectra

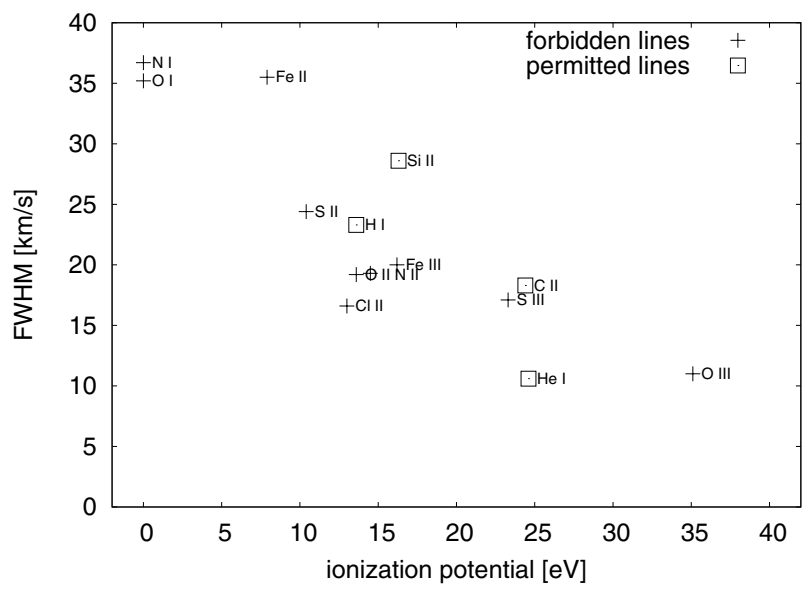

Fig. 3. Deconvolved line widths vs. ionization potentials.

obtained before, due to light loss on the slit. This could affect the observed [O III] $5007 \AA$ to $\mathrm{H} \beta$ line flux ratio.

The spectrum published by Escudero et al. (2004) was obtained with a 2 arcsec wide slit, which did not resolve the nebula. The SALT spectrum was obtained with a 1 arcsec wide slit, which could resolve the nebula, but only under very good seeing conditions. However, the seeing during the SALT observation was about 1.3 arcsec, a value that is comparable to the extent of the nebula. The seeing during the $1.9 \mathrm{~m}$ Radcliffe telescope observations, which were obtained in the same year, varied between 1.3 and 1.9 arcsec. The SALT and $1.9 \mathrm{~m}$ Radcliffe telescope spectra show a consistent [O III] $5007 \AA$ flux, a value that is higher than in the spectrum by Escudero et al. (2004) and also higher than in the VLT spectrum, which was obtained with the most narrow slit of all the observations used in this work. This confirms that the flux increase is real.

The nebular spectrum is rich in [Fe II] and [Fe III] lines (Table 4). The [Fe II], [O I], [N I] and permitted O I lines show wide profiles with sharp edges in the VLT spectrum. They can be modelled with a shell expanding with a velocity of about $20 \mathrm{~km} \mathrm{~s}^{-1}$. [Fe III], [O II], and Si II lines show slighlty asymmetric and narrower profiles (Fig. 2). Other nebular lines show symmetric profiles.

The line widths for different ions against the ionization potential are presented in the Fig. 3. The ions of higher ionization potential show lower expansion velocity than the ions of lower ionization potential. As the high excitation lines form closer to the central star than the low excitation lines, the observed trend indicates the existence of a strong velocity gradient across the nebular shell.

Stellar absorptions of H, He I, and He II are present in the VLT spectrum. Other stellar lines include O III and Si IV absorptions. No stellar wind lines were observed.

\subsection{Torun model}

The object Hen 2-260 is obviously a bipolar nebula; however, it is not extremely elongated. We think of it as composed of a barrel-shaped main body with moderate extensions (low density lobes) in the axial direction (which is nearly in the plane of the sky). We propose to approximate this PN with a spherical model, which certainly corresponds to the dominating dense barrel. We expect that the lobes contribute a little to the total line fluxes, and by considering the low spatial resolution of spectroscopy, we expect that skipping the lobes is not a bad approximation. 

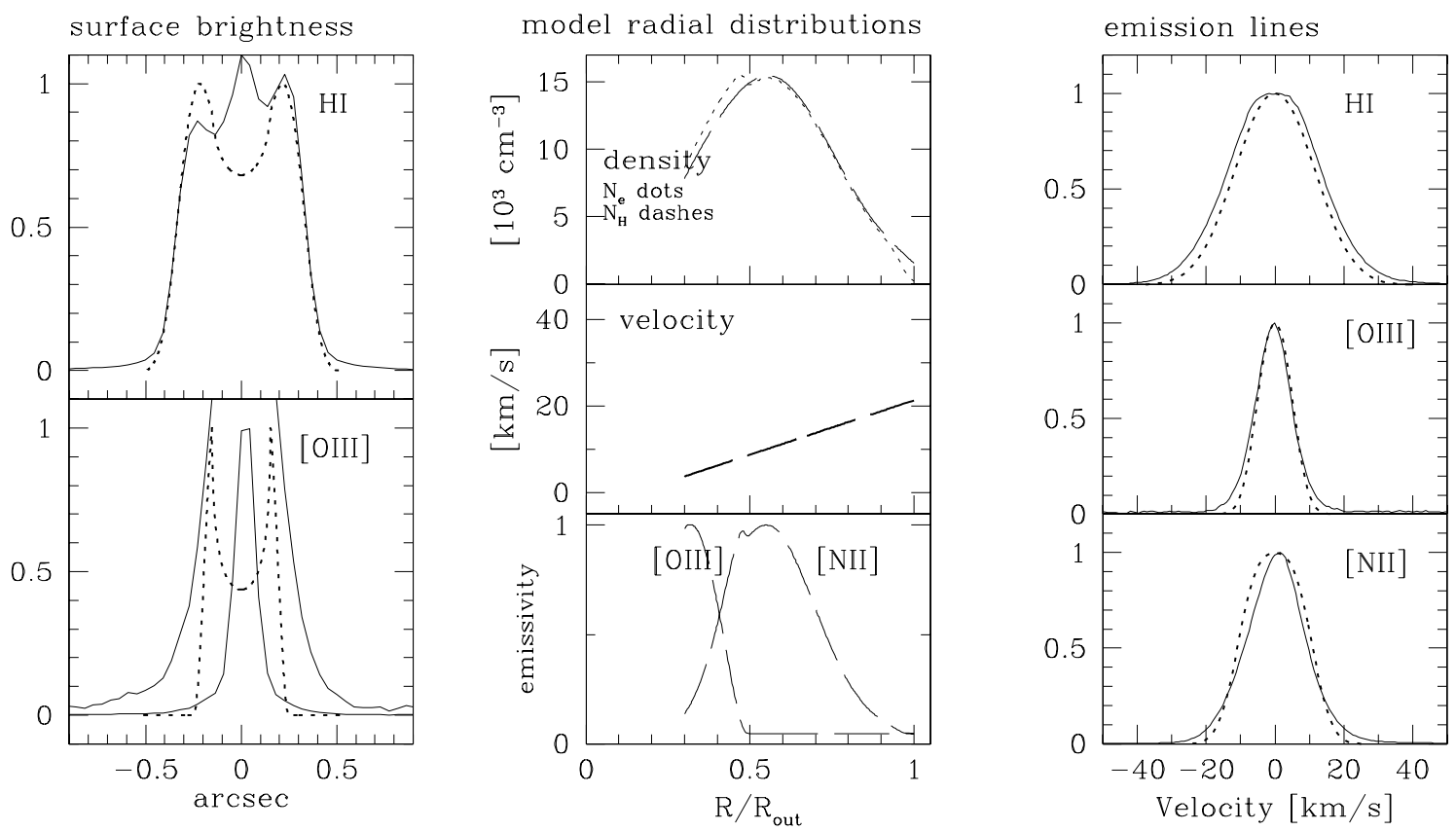

Fig. 4. Torun model results for a stellar temperature of $25200 \mathrm{~K}$ and a luminosity of $1400 \mathrm{~L}_{\odot}$. The observed data are drawn in solid lines, while model fits and model parameters are shown as dotted and dashed lines. The "surface brightness" panel (left) shows the slices through the H $\alpha$ and [O III] $5007 \AA$ image centre taken along a nebular short axis with the model brightness profile superimposed. Both slices are scaled to unity; however, the [O III] image is additionally shown with different scaling (see text) because the centre is dominated by the stellar flux. The radial distributions of the best-fit model for selected parameters are shown next. The right-most panel shows the observed and modelled profiles of three nebular lines: $\mathrm{H} \alpha$, [O III] $5007 \AA ̊$, and [N II] $6584 \AA$.

We applied the Torun photo-ionization codes to de-compose the structure of the nebula (Gesicki \& Zijlstra 2003). The star is assumed to be a black body. The nebula is approximated as a spherical shell with a radial density distribution that is similar to a reversed parabola and a velocity field that linearly increases with radius. Locating the best-fit parameters is aided by a routine with a genetic algorithm.

The code calculates 1-dimensional (radial) emissivity profiles. Assuming spherical symmetry, they are converted to the observed projected intensity profiles. These profiles are compared with the slices along the minor axis of the HST images, which are averaged over 9 pixels. The model density distribution is manually modified at the maximum, the inner radius and outer radius to obtain a best similarity (judged visually) between both images.

With the derived density profile, a genetic algorithm searches for the stellar parameters and for the nebular ionised mass that best fit the line flux ratios. Later, the profiles of lines with different ionization states, tracing different radii within the nebula are fitted to obtain the radial velocity profile within the nebula.

It was relatively straightforward to find a density profile that reproduced the $\mathrm{H} \alpha$ slice down to the level of $10 \%$ of the maximum intensity (Fig. 4). The low density outer regions could be, in principle, extended further out to reproduce the halo; however, the PN is more complicated than a sphere, so we therefore did not go further with fine-tuning of the density distribution. The density contrast between the maximum and the halo is around 10 . The [O III] line is very weak in Hen 2-260, so we see mainly the very strong stellar continuum in the HST image. The scaled-to-unity image certainly is not appropriate to compare with the model. We show the [O III] image scaled accordingly to its strengths relative to $\mathrm{H} \alpha$ in Fig. 4. The nebular emission is well localised in the lowest intensity area.
The Torun model takes a limited number of most representative emission lines into account. They are taken from SALT spectrum analysed in this paper. The automated search routine found this fit for a stellar temperature of $25200 \mathrm{~K}$ and a luminosity of $1400 L_{\odot}$ with nebular ionised mass of $0.01 M_{\odot}$.

\subsection{Cloudy modelling}

We computed a grid of models to fit the observed fluxes using Cloudy c10.01, as described by Ferland et al. (2013). The distance of $12 \mathrm{kpc}$ was adopted. We used a spherical model of the nebula with a constant density. Central star radiation was modelled with TLUSTY model atmospheres (Hubeny \& Lanz 1995). The stellar luminosity, temperature, inner radius, nebular density, and elemental and grain abundances were varied to find the best solution (van Hoof \& van de Steene 1999). The model used silicate grains. We used the $90 \mu \mathrm{m}$ flux as a stopping criterion.

Initially, we did not include the full list of the singly and doubly ionised iron transitions. We added the iron lines to the model after the solution converged. All the observational constraints used are given in the Table 2 . The full list of line fluxes obtained from the SALT spectrum and modelled with the Cloudy is given in the Table 3.

There is a severe discrepancy in the elemental abundances obtained by other authors and us (Table 2). Chiappini et al. (2009) report large uncertainties for the determination of nebular abundances for Hen 2-260 due to poorly known ionizing correction factors for this object. The $V$-band magnitude of 13.4 (assuming the distance of $12 \mathrm{kpc}$ ) is a bit fainter than the observed brightness (Fig. 1), taking the extinction into account. Some differences between the observed and modelled fluxes for individual lines exceed the observational uncertainties, but all the diagnostic line ratios agree very well with the ratios predicted by the model. 
Table 2. A list of observed/modelled parameters compared to the results of our Cloudy model of Hen 2-260.

\begin{tabular}{ccc}
\hline \hline & Other authors & This paper \\
\hline$F_{\text {WISE1 } 1.6 \mu \mathrm{m}[\mathrm{mJy}]}$ & $640 \pm 9$ & 610 \\
$F_{\text {WISE22.1 } \mu \mathrm{m}[\mathrm{mJy}]}$ & $7540 \pm 50$ & 6820 \\
$F_{\text {IRAS12 } \mu \mathrm{m}[\mathrm{mJy}]}$ & $590 \pm 60$ & 649 \\
$F_{\text {IRAS25 } \mu \mathrm{m}[\mathrm{mJy}]}$ & $8100 \pm 500$ & 7370 \\
$F_{\text {IRAS60 } \mu \mathrm{m}[\mathrm{mJy}]}$ & $2900 \pm 300$ & 3322 \\
$F_{\text {IRAS100 } \mu \mathrm{m}[\mathrm{mJ}]}$ & $<4220$ & \\
$F_{\text {AKARI65 } \mu \mathrm{m}[\mathrm{mJy}]}$ & 1399 & \\
$F_{\text {AKARI90 } \mu \mathrm{m}[\mathrm{mJ}]}$ & $1167 \pm 112$ & 1167 \\
$F_{\text {AKARI160 } \mu \mathrm{mJy}]}[\mathrm{mJy}]$ & 210.3 & \\
$F_{1.4 \mathrm{GHz}}[\mathrm{mJy}]$ & $8.1 \pm 0.5^{a}$ & \\
$F_{5 \mathrm{GHz}}[\mathrm{mJy}]$ & $13^{b}$ & 12.47 \\
$\log F(\mathrm{H} \alpha)\left[\mathrm{erg} \mathrm{cm}{ }^{-2} \mathrm{~s}^{-1}\right]$ & $-11.26 \pm 0.04^{e}$ & $-11.19 \pm 0.03$ \\
$\log F(\mathrm{H} \beta)\left[\mathrm{erg} \mathrm{cm}{ }^{-2} \mathrm{~s}^{-1}\right]$ & $-12.13 \pm 0.1^{h}$ & \\
$\log \left(L / L_{\odot}\right)$ & $3.79^{c}$ & 3.11 \\
$\in(\mathrm{N})$ & $4.09_{-0.14}^{+0.15}(3.8 \pm 0.26)^{d}$ & \\
$\in(\mathrm{O})$ & $7.14^{f}, 7.24^{g}$ & 7.65 \\
$\in(\mathrm{S})$ & $8.18^{f}, 8.00^{g}$ & 8.69 \\
$\in(\mathrm{Ar})$ & $6.29^{f}, 6.22^{g}$ & 6.70 \\
$\log \left(r_{\mathrm{in}}\right)[\mathrm{cm}]$ & $6.97^{f}, 5.33^{g}$ & 5.99 \\
$\log \left(r_{\text {out }}\right)[\mathrm{cm}]$ & & 16.83 \\
$\log n_{\mathrm{H}}\left[\mathrm{cm}{ }^{-3}\right]$ & & 17.20 \\
$T_{\text {eff }}[\mathrm{K}]$ & $28000^{i}$ & 3.43 \\
\hline
\end{tabular}

Notes. ${ }^{(a)}$ Condon \& Kaplan (1998); ${ }^{(b)}$ Aaquist \& Kwok (1990); ${ }^{(c)}$ for the adopted distance of $12 \mathrm{kpc}$, Gesicki \& Zijlstra (2007). ${ }^{(d)}$ for the adopted distance of $8 \mathrm{kpc}$, Hultzsch et al. (2007); ${ }^{(e)}$ Frew et al. (2013); (f) Escudero et al. (2004); ${ }^{(g)}$ Chiappini et al. (2009); ${ }^{(h)}$ Acker et al. (1992); ${ }^{(i)}$ Hultzsch et al. (2007).

After the Cloudy models converged to the best solution, we ran a grid of models for different temperatures, which ranged from 34.6 to $35.8 \mathrm{kK}$ to fit the temporal evolution of the [O III] $5007 \AA$ line flux. We have chosen this line because it is not affected by stellar absorptions, it is stronger than other high excitation lines, and it is very close to the $\mathrm{H} \beta$ line, so the flux ratio of both lines is not very sensitive to the wavelength-dependent calibration errors, such as atmospheric extinction correction or differential atmospheric refraction. We also scaled the nebular density and inner radius, assuming a constant expansion rate.

The observed [O III] $5007 \AA$ flux was best modelled with the stellar temperature of $34875 \pm 91 \mathrm{~K}$ in $2001,34944 \pm 20 \mathrm{~K}$ in 2005 , and $35289 \pm 52 \mathrm{~K}$ in 2012 . The temperature uncertainties correspond only to the standard deviation of the [O III] $5007 \AA$ line flux. Systematic error is larger, but it is similar in all the temperature determinations and is cancelled out when computing the difference between the temperatures. Thus, the heating rate can be firmly established.

The observed heating rate of $45 \pm 7 \mathrm{Kyr}^{-1}$ can be modelled with the post-AGB evolutionary track that is interpolated for the final mass of $0.626_{-0.005}^{+0.003} M_{\odot}\left(0.626_{-0.010}^{+0.007} M_{\odot}\right)$ by using the models published by Blöcker (1995) or $0.645_{-0.008}^{+0.008} M_{\odot}\left(0.645_{-0.016}^{+0.015} M_{\odot}\right)$ by using Vassiliadis \& Wood (1993) at a one (two) sigma confidence level (Fig. 5).

The mass errors correspond to the heating rate uncertainty. The mass of the star is very close to the peak of the mass distribution for central stars of planetary nebulae of $0.61 M_{\odot}$ (Gesicki $\&$ Zijlstra 2007). The age of the central, spherical component of the nebula of the order of 1000 years (for a distance of $12 \mathrm{kpc}$, $r_{\text {out }}=0.25 \operatorname{arcsec}$ and $V_{\exp }=20 \mathrm{~km} \mathrm{~s}^{-1}$ ) is in better agreement
Table 3. Observed, dereddened, and modelled line fluxes of Hen 2-260 obtained with the SALT and $1.9 \mathrm{~m}$ Radcliffe telescopes that are normalised relative to $F(\mathrm{H} \beta)=100$.

\begin{tabular}{|c|c|c|c|c|}
\hline$\lambda_{\text {lab }}[\AA]$ & Ion & Observed & Dereddened & Modelled \\
\hline $3726.03+3728.82$ & [O II] & 69.1 & 109.7 & 120.5 \\
\hline 3835.38 & $\mathrm{HI}$ & 3.7 & 5.7 & 7.8 \\
\hline 3889.05 & $\mathrm{HI}$ & 6.9 & 10.2 & 10.6 \\
\hline 3970.07 & $\mathrm{HI}$ & 10.2 & 14.6 & 16.3 \\
\hline 4068.60 & [S II $]$ & 4.5 & 6.2 & 5.3 \\
\hline 4076.01 & {$[\mathrm{~S} \mathrm{II}]$} & 2.4 & 3.3 & 1.8 \\
\hline 4101.74 & $\mathrm{HI}$ & 17.0 & 23.1 & 27.2 \\
\hline 4340.46 & H I & 36.4 & 45.3 & 48.6 \\
\hline 4658.05 & [Fe III $]$ & 2.00 & 2.19 & 0.51 \\
\hline 4701.54 & {$[\mathrm{Fe} \mathrm{III}]$} & 0.88 & 0.94 & 0.22 \\
\hline 4733.90 & {$[\mathrm{Fe}$ III $]$} & 0.37 & 0.39 & 0.10 \\
\hline 4754.69 & {$[\mathrm{Fe} \mathrm{III}]$} & 0.34 & 0.35 & 0.09 \\
\hline 4769.43 & {$[\mathrm{Fe}$ III $]$} & 0.24 & 0.25 & 0.07 \\
\hline 4777.68 & {$[\mathrm{Fe} \mathrm{III}]$} & 0.18 & 0.19 & 0.05 \\
\hline 4814.53 & {$[\mathrm{Fe} \mathrm{II}]$} & 0.18 & 0.18 & \\
\hline 4861.33 & H I & 100.00 & 100.00 & 100.00 \\
\hline 4881.00 & {$[\mathrm{Fe}$ III $]$} & 1.15 & 1.14 & 0.27 \\
\hline 4930.54 & [Fe III] & 0.13 & 0.13 & 0.03 \\
\hline 4958.91 & [O III] & 2.41 & 2.33 & 2.23 \\
\hline 4987.21 & {$[\mathrm{Fe} \mathrm{III}]$} & 0.12 & 0.11 & 0.05 \\
\hline 5006.84 & [O III] & 7.85 & 7.45 & 6.71 \\
\hline 5015.68 & He I & 0.50 & 0.47 & 0.45 \\
\hline 5041.02 & Si II & 0.28 & 0.26 & \\
\hline 5055.98 & Si II & 0.48 & 0.44 & \\
\hline 5084.77 & [Fe III] & 0.07 & 0.06 & 0.02 \\
\hline 5146.58 & OI & 0.19 & 0.17 & \\
\hline 5158.78 & {$[\mathrm{Fe} \mathrm{II}]$} & 0.38 & 0.33 & \\
\hline 5197.90 & {$[\mathrm{~N} \mathrm{I}]$} & 0.36 & 0.31 & 0.21 \\
\hline 5261.62 & [Fe II] & 0.22 & 0.18 & \\
\hline 5270.40 & [Fe III] & 1.54 & 1.30 & 0.31 \\
\hline 5299.01 & OI & 0.25 & 0.21 & \\
\hline 5333.65 & [Fe II] & 0.10 & 0.08 & \\
\hline 5512.73 & OI & 0.18 & 0.14 & \\
\hline 5517.72 & [Cl IIII $]$ & 0.07 & 0.06 & 0.05 \\
\hline 5527.61 & {$[\mathrm{Fe}$ II $]$} & 0.07 & 0.05 & \\
\hline 5537.89 & [Cl III] & 0.20 & 0.15 & 0.13 \\
\hline 5554.83 & OI & 0.22 & 0.16 & \\
\hline 5695.19 & C III & 0.21 & 0.15 & \\
\hline 5754.65 & [N II] & 4.19 & 3.02 & 2.91 \\
\hline 5875.66 & He I & 2.89 & 2.03 & 2.31 \\
\hline 5958.51 & $\mathrm{OI}_{\mathrm{I}}$ & 0.40 & 0.27 & \\
\hline 5978.93 & Si II & 0.24 & 0.16 & \\
\hline 6046.39 & OI & 0.54 & 0.36 & \\
\hline 6300.30 & [O I] & 3.38 & 2.10 & 2.14 \\
\hline 6312.10 & [S III] & 1.49 & 0.92 & 1.28 \\
\hline 6347.09 & Si II & 0.57 & 0.35 & \\
\hline 6363.78 & [O I $]$ & 1.12 & 0.68 & 0.68 \\
\hline 6371.38 & Si II & 0.32 & 0.19 & \\
\hline 6548.10 & [N II] & 62.87 & 36.23 & 38.86 \\
\hline 6562.77 & $\mathrm{HI}$ & 493.25 & 283.24 & 282.50 \\
\hline 6583.50 & [N II] & 185.90 & 106.22 & 114.68 \\
\hline 6666.80 & [Ni II] & 0.08 & 0.05 & \\
\hline 6678.15 & He I & 0.80 & 0.45 & 0.63 \\
\hline 6716.44 & [S II] & 3.58 & 2.00 & 1.66 \\
\hline 6730.82 & [S II] & 8.00 & 4.46 & 3.67 \\
\hline 7002.21 & O I & 0.72 & 0.38 & \\
\hline 7065.25 & He I & 1.92 & 1.00 & 0.77 \\
\hline 7135.8 & [Ar III] & 2.29 & 1.17 & 1.22 \\
\hline 7155.16 & [Fe II] & 0.73 & 0.37 & \\
\hline 7254.53 & O I & 0.81 & 0.41 & \\
\hline 7319.46 & [O II] & 49.78 & 24.27 & 21.86 \\
\hline 7330.13 & [O II] & 40.44 & 19.67 & 17.72 \\
\hline 7377.83 & [Ni II] & 0.90 & 0.44 & \\
\hline 7388.18 & [Fe II] & 0.19 & 0.09 & \\
\hline 7411.61 & [Ni II] & 0.16 & 0.08 & \\
\hline
\end{tabular}




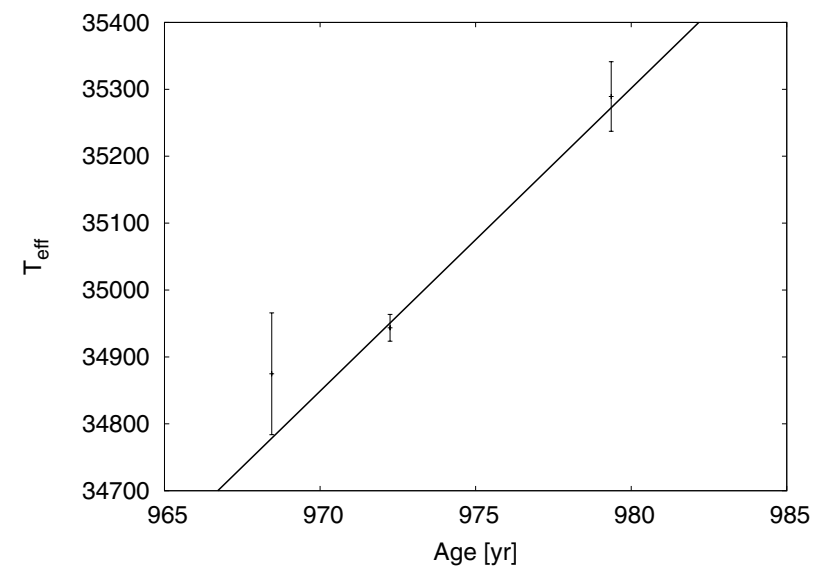

Fig. 5. Observed temperature evolution of the central star. The solid line represents the evolutionary track for the interpolated model with a final mass of $0.626 M_{\odot}$ (Blöcker 1995).

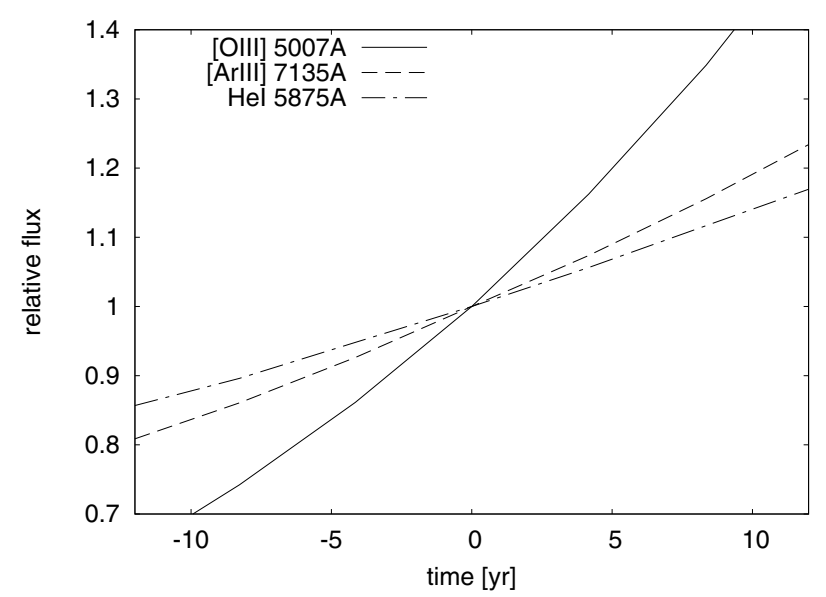

Fig. 6. Predicted evolution of three line fluxes. The zero point on the time axis corresponds to 2012 (SALT observation of the He 2-260).

with the post-AGB evolutionary track value given by Blöcker (980 years) than Vassiliadis \& Wood ( $480 \mathrm{yr}$ ). However, the age of the object determined from the evolutionary tracks depends on the limited accuracy of the absolute temperature determination of the star. If we adopted $28000 \mathrm{~K}$ instead of about $35000 \mathrm{~K}$, which is modelled with Cloudy, then the derived age would be reduced by about $200 \mathrm{yr}$. On the other hand, the age depends on the adopted zero scale by the evolutionary models.

The Cloudy models predict a $16 \%$ flux increase for the He I $5875 \AA$ line, a $13 \%$ increase for the He I $6678 \AA$ line, a $20 \%$ increase for the He I $7065 \AA$ line, and a $18 \%$ increase for the [Ar III] $7135 \AA$ line between 2001 and 2012 (Fig. 6). We observe a faster flux increase for those lines. However, those lines are fainter than the [O III] $5007 \AA$ line, and some of them can be affected by the stellar absorptions, so the flux change determination is less reliable.

A significant systematic error may affect the determination of the absolute central star temperature. However, the heating rate depends primarily on the central star mass and does not change steeply during the evolution of a star between 20000 and $140000 \mathrm{~K}$ (Fig. 7). The mass derivation based on the heating rate does not depend on the absolute age of the nebula, unlike the dynamical method used by Gesicki \& Zijlstra (2007).

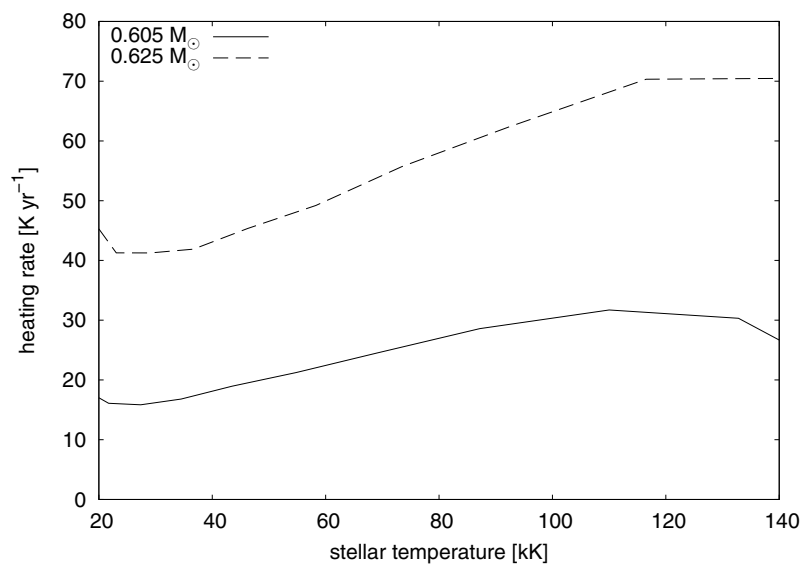

Fig. 7. Modelled evolution of the heating rate of central stars for two different masses (Blöcker 1995).

\subsection{Optical photometry}

The central star of the PN Hen 2-260 shows photometric variability on a timescale of hours or days (Fig. 8). Photometry taken at individual nights cannot be clearly seen in the bottom of Fig. 8 due to large time range of the observations shown. Two best sampled individual nights are plotted at the top of Fig. 8. The observations do not indicate sinusoidal variations. The amplitude of the variability is of the order of $0.1 \mathrm{mag}$ in the $I$ band. However, the real amplitude may be somewhat higher, since the nebular contribution was not removed.

No single dominant periodicity can be identified in the power spectrum (Fig. 9). The power spectrum is noisy; however, two strongest peaks can be identified at 0.68 and $1.68 \mathrm{~d}^{-1}$. The latter one may be the alias of the former. Another peak is present at $1.06 \mathrm{~d}^{-1}$.

The photometric data were taken non-uniformly during different seeing conditions. We cannot draw firm conclusions regarding the nature of the photometric variability of Hen 2-260. It could be caused by pulsations, magnetic activity of the star, binarity, or a combination of those.

We do not detect any emission lines originating from the stellar wind in the VLT spectrum; thus, the variability of the central star can be most likely attributed to stellar pulsations. Our object fulfills the criteria of a group of variable CSPNs (Handler 2003): the temperature between 25 and $50 \mathrm{kK}$, (semi)regular variability on a timescale of several hours and hydrogen-rich spectra. However, more uniform coverage that is compatible with the variability timescale of the object would be needed to constrain the nature of the variability.

\section{Discussion}

The spectra of most of PNe are usually observed only once in a lifetime. Most of the PNe included in the largest catalogue of the PN fluxes to date, which are provided by Acker et al. (1992), have never been observed again. Our results show that monitoring of nebular fluxes may be used as an excellent tool to study the evolution of central stars of PNe. Spectroscopic observations of a PN that cover a time span of only a decade can already reveal the temperarure change of its central star. The derived heating rate of central stars is steeply dependent on the mass of the star. The comparison of the heating rate with the post-AGB evolutionary models can provide precise mass determinations.

We discovered a 50\% increase of the [O III] $5007 \AA$ emission line flux in a period of eleven years in Hen 2-260. The pace of 

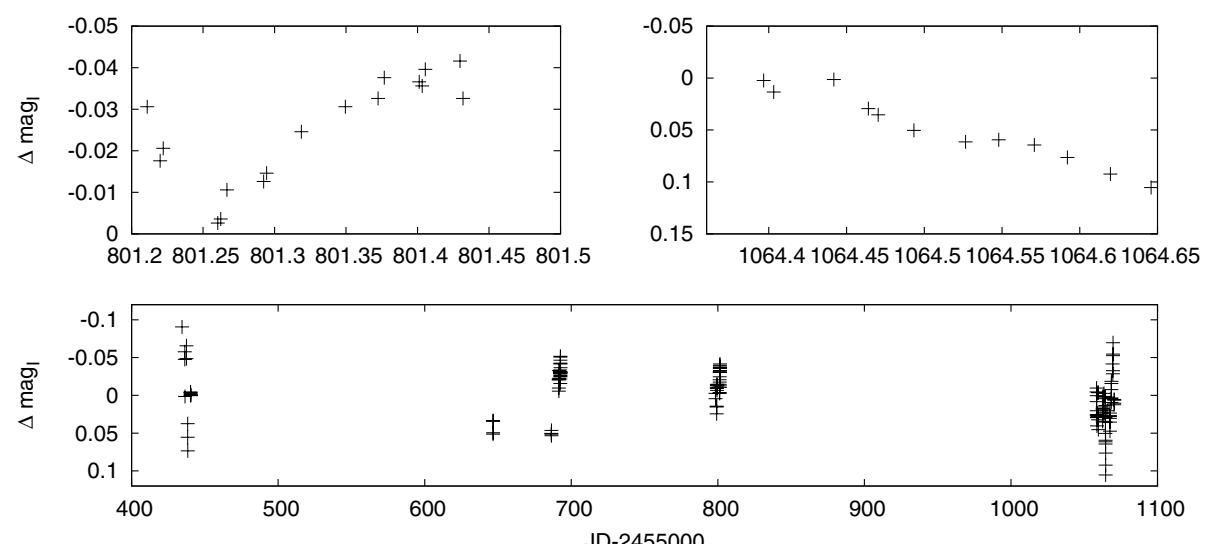

Fig. 8. SAAO I band 2010-2012 photometry of Hen 2-260. Photometry taken on two separate nights is shown above the light curve collected between 2010 and 2012 .

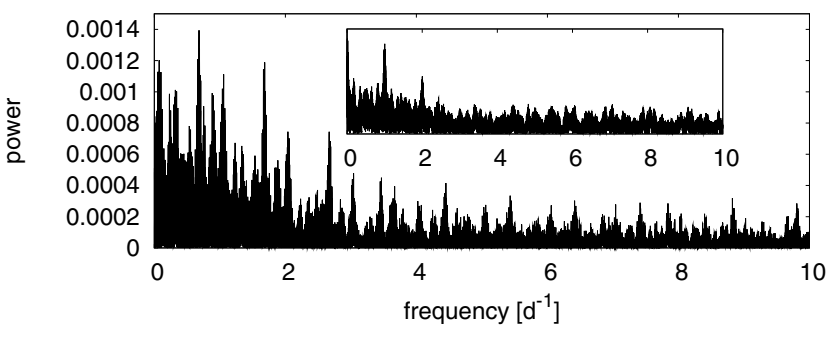

Fig. 9. Power spectrum of $I$-band observations of Hen 2-260 along with the power window shown in the inset.

the evolution of the central star and the kinematical age of the PN are consistent with the existing evolutionary models. Further observations will allow us to determine the heating rate and mass of the star more accurately.

Acknowledgements. This work was financially supported by NCN of Poland through grants Nos. 2011/01/D/ST9/05966, 2011/03/B/ST9/02552, N N203 511838 and 719/N-SALT/2010/0. PvH acknowledges support from the Belgian Science Policy office through the ESA PRODEX program. This paper uses observations made at the South African Astronomical Observatory (SAAO). Some of the observations reported in this paper were obtained with the Southern African Large Telescope (SALT), proposal 2011-3-POL-002, P.I. M. Hajduk. We made use of the Atomic Line List available at http://www.pa.uky.edu/ $\sim$ peter/newpage/

\section{References}

Aaquist, O. B., \& Kwok, S. 1990, A\&AS, 84, 229

Acker, A., Raytchev, B., Koeppen, J., \& Stenholm, B. 1991, A\&AS, 89, 237

Acker, A., Marcout, J., Ochsenbein, F., et al. 1992, The Strasbourg-ESO Catalogue of Galactic Planetary Nebulae. Parts I, II (ESO)

Arkhipova, V. P., Ikonnikova, N. P., Kniazev, A. Y., \& Rajoelimanana, A. 2013, Astron. Lett., 39, 201

Blöcker, T. 1995, A\&A, 299, 755
Chiappini, C., Górny, S. K., Stasińska, G., \& Barbuy, B. 2009, A\&A, 494, 591 Condon, J. J., \& Kaplan, D. L. 1998, ApJS, 117, 361

Dudziak, G., \& Walsh, J. R. 1997, in The 1997 HST Calibration Workshop with a New Generation of Instruments, eds. S. Casertano, R. Jedrzejewski, T. Keyes, \& M. Stevens, 338

Escudero, A. V., Costa, R. D. D., \& Maciel, W. J. 2004, A\&A, 414, 211

Feibelman, W. A., Aller, L. H., \& Hyung, S. 1992, PASP, 104, 339

Ferland, G. J., Porter, R. L., van Hoof, P. A. M., et al. 2013, Rev. Mex. Astron. Astrofis., 49, 137

Fitzpatrick, E. L. 1999, PASP, 111, 63

Frew, D. J., Bojičić, I. S., \& Parker, Q. A. 2013, MNRAS, 431, 2

Gautschy, A. 1993, MNRAS, 265, 340

Gesicki, K., \& Zijlstra, A. A. 2003, MNRAS, 338, 347

Gesicki, K., \& Zijlstra, A. A. 2007, A\&A, 467, L29

Górny, S. K., Perea-Calderón, J. V., García-Hernández, D. A., García-Lario, P., \& Szczerba, R. 2010, A\&A, 516, A39

Guzman-Ramirez, L., Zijlstra, A. A., Níchuimín, R., et al. 2011, MNRAS, 414, 1667

Handler, G. 2003, in Planetary Nebulae: Their Evolution and Role in the Universe, eds. S. Kwok, M. Dopita, \& R. Sutherland, IAU Symp., 209, 237

Hubeny, I., \& Lanz, T. 1995, ApJ, 439, 875

Hultzsch, P. J. N., Puls, J., Méndez, R. H., et al. 2007, A\&A, 467, 1253

Krist, J. E., Hook, R. N., \& Stoehr, F. 2011, in Proc. SPIE, 8127, 0J

Lenz, P., \& Breger, M. 2005, Commun. Asteroseismol., 146, 53

Nahar, S. N., \& Pradhan, A. K. 1996, A\&AS, 119, 509

Perea-Calderón, J. V., García-Hernández, D. A., García-Lario, P., Szczerba, R., \& Bobrowsky, M. 2009, A\&A, 495, L5

Pottasch, S. R. 1984, Planetary nebulae - A study of late stages of stellar evolution, Astrophys. Space Sci. Lib. (Dordrecht: D. Reidel Publishing Co), vol. 107

Pottasch, S. R., \& Bernard-Salas, J. 2013, A\&A, 550, A35

Quinet, P., Le Dourneuf, M., \& Zeippen, C. J. 1996, A\&AS, 120, 361

Schwarzenberg-Czerny, A. 1996, ApJ, 460, L107

Stanghellini, L., \& Haywood, M. 2010, ApJ, 714, 1096

Storey, P. J., \& Zeippen, C. J. 2000, MNRAS, 312, 813

Van de Steene, G. C., \& Zijlstra, A. A. 1994, A\&AS, 108, 485

van Hoof, P. A. M., \& van de Steene, G. C. 1999, MNRAS, 308, 623

Vassiliadis, E., \& Wood, P. R. 1993, ApJ, 413, 641

Zalewski, J. 1993, Acta Astron., 43, 431

Zhang, C. Y. 1995, ApJS, 98, 659

Zijlstra, A. A., van Hoof, P. A. M., \& Perley, R. A. 2008, ApJ, 681, 1296 